\title{
Sensibilidade de isolados de fungos entomopatogênicos às radiações solar, ultravioleta e à temperatura
}

\author{
Sensibility of isolates of entomopathogenic fungi to solar radiation, \\ ultraviolet rays and temperature
}

\author{
Manuela Teodoro de Oliveira ${ }^{1}$, Antonio Carlos Monteiro ${ }^{1 *}$, Newton La Scala Júnior ${ }^{2}$, \\ José Carlos Barbosa², Dinalva Alves Mochi ${ }^{1}$
}

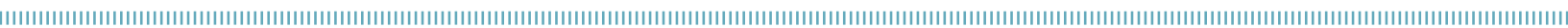

RESUMO: Este trabalho teve por objetivo avaliar a sensibilidade de isolados dos fungos Metarhizium anisopliae (Metsch.) Sorok. e Beauveria bassiana (Bals). Vuill. ao efeito das radiaçóes solar e ultravioleta e da temperatura. Conídios dos isolados foram expostos, por vários períodos, aos raios de um simulador solar em diversas irradiâncias e a uma lâmpada de raios ultravioleta germicida. Os conídios do isolado de $M$. anisopliae foram também expostos às temperaturas de 19,$5 ; 24,2$ e $31,0^{\circ} \mathrm{C}$, e os do isolado de $B$. bassiana a 19,4 ; 20,8 e $28,3^{\circ} \mathrm{C}$, e 18,$7 ; 23,8$ e $30,9^{\circ} \mathrm{C}$. Avaliou-se a germinação de conídios pelo teste de viabilidade. Os isolados dos fungos se mostraram bastantes sensíveis aos raios do simulador solar e aos raios ultravioleta. A germinação de ambos sofreu significativa redução a partir de 30 minutos de exposição à radiação do simulador solar. $\mathrm{O}$ efeito mais severo foi evidenciado pelo isolado de B. bassiana, com grande redução da germinação dos conídios em todas as irradiâncias testadas. A sensibilidade à radiaçáo ultravioleta também foi grande, pois ocorreu acentuada redução da germinação dos conídios do isolado de $M$. anisopliae (38,2\%) e de B. bassiana (65\%) já aos 30 segundos de exposição. A temperatura afetou a viabilidade de ambos os fungos. Temperaturas entre 23,8 e $31^{\circ} \mathrm{C}$ favoreceram a germinação dos conídios, enquanto temperaturas próximas de $20^{\circ} \mathrm{C}$ dificultaram a germinação.

PALAVRAS-CHAVE: Beauveria bassiana; Metarhizium anisopliae; controle biológico; exposição à luz.

\begin{abstract}
This study aimed to access the sensibility of isolates of the fungus Metarhizium anisopliae (Metsch.) Sorok. and Beauveria bassiana (Bals.) Vuill. to the effect of solar and ultraviolet radiation and temperature. Conidia were exposed for various periods to the rays from a solar simulator at various irradiances, and to light germicidal ultraviolet rays. Conidia of the isolate of $M$. anisopliae were also exposed to temperatures of $19.5,24.2$ and $31.0^{\circ} \mathrm{C}$ and the isolate of $B$. bassiana to $19.4,20.8$ and $28.3^{\circ} \mathrm{C}$, and also to $18.7,23.8$ and $30.9^{\circ} \mathrm{C}$. The germination of conidia was evaluated by the viability test. The fungal isolates showed to be very sensitive to the solar simulator and ultraviolet rays. Germination of both was significantly decreased starting from 30 minutes of exposure to the rays of the solar simulator. The most severe effect was evidenced by the isolate of $B$. bassiana with great reduction in conidia germination in all the tested irradiances. Sensitivity to ultraviolet radiation was also great, showing a marked reduction in the germination of $M$. anisopliae (38.2\%) and B. bassiana $(65 \%)$ conidia after 30 seconds of exposure. The temperature affected the viability of both fungi. Temperatures ranging of 23.8 to $31^{\circ} \mathrm{C}$ favor the germination of conidia while temperatures around $20^{\circ} \mathrm{C}$ constrained germination.
\end{abstract}

KEYWORDS: Beauveria bassiana; Metarhizium anisopliae; biological control; light exposure. 


\section{INTRODUÇÃO}

Os fungos entomopatogênicos são considerados importantes agentes para o controle biológico de pragas (INGLIs et al., 2008). Entre os mais utilizados se encontram o Metarbizium anisopliae e Beauveria bassiana (Alves, 1998).

M. anisopliae ganhou especial atenção dos pesquisadores brasileiros quando foi observada sua ocorrência epizoótica sobre Mahanarva posticata (Stal) (Hemiptera: Cercopidae), a cigarrinha da folha da cana-de-açúcar, resultando em conhecido programa de utilizaçáo do fungo para o controle dessa praga (Alves, 1998). Com a expansão do sistema de colheita mecanizada da cana-de-açúcar, vários trabalhos (Almeida et al., 2004; Dinardo-Miranda et al., 2004) mostraram que o fungo pode ser utilizado com sucesso no controle da cigarrinha-da-raíz Mahanarva fimbriolata (Stal) (Hemiptera: Cercopidae).

Devido à sua distribuição cosmopolita e frequente presença na natureza, Beauveria bassiana é um dos mais encontrados e reconhecidos fungos patógenos de insetos (Rehner, 2005). No Brasil usam-se aplicaçóes do fungo para o controle de pragas de culturas importantes como a broca-do-cafeeiro Hypothenemus hampei Ferrari (Coleoptera: Scolytidae) (Neves et al., 2010) e a broca-da-bananeira Cosmopolites sordidus Germar (Coleoptera: Curculionidae) (Batista Filho et al., 2010).

Quando aplicados no campo, os entomopatógenos estão sujeitos à açáo de fatores bióticos e abióticos, que podem influenciar sua sobrevivência, propagação e infecção no hospedeiro. Entre os abióticos, destaca-se a radiação solar, que é um dos principais fatores ambientais capazes de diminuir o tamanho das populaçóes de fungos e/ou reduzir a disseminação de algumas espécies no ambiente (Wraight et al., 2007).

A radiação ultravioleta (UV) é um dos maiores problemas ambientais que afetam esses fungos, podendo provocar danos diretos e indiretos que reduzem sua eficiência contra os insetos, atuando na germinação dos conídios e sobre os estágios iniciais do tubo germinativo (Braga et al., 2001a, 2001b, 2001c; 2002). Os danos diretos incluem a inativação dos conídios, danos letais ao DNA e mutaçôes. Entre os indiretos estão o aquecimento e a dessecação dos conídios (Nicholson et al., 2000).

Outro fator importante para a sobrevivência dos fungos entomopatogênicos no campo é a temperatura. Altas temperaturas prejudicam a sobrevivência do fungo, ao passo que baixas temperaturas aumentam sua persistência, característica essa desejável (Rath, 2002). Temperaturas maiores do que $30^{\circ} \mathrm{C}$ dificultam o crescimento e a sobrevivência de $M$. anisopliae no solo, enquanto temperaturas medianas (21 a $27^{\circ} \mathrm{C}$ ) favorecem o crescimento e a sobrevivência do fungo (LANZA et al., 2009).

A eficiência de fungos entomopatogênicos no controle de insetos-praga depende, entre outros aspectos, de sua sobrevivência e atividade em condiçóes compatíveis. Temperatura e intensidade de radiação são fatores abióticos bastante variáveis e seus efeitos sobre as populaçôes desses fungos no ambiente necessitam ser melhor conhecidos. Assim sendo, este trabalho objetivou avaliar a sensibilidade de isolados dos fungos $M$. anisopliae e B. bassiana ao efeito das radiações solar e ultravioleta e da temperatura.

\section{MATERIAL E MÉTODOS}

Foram utilizados os isolados JAB 68 de M. anisopliae, obtido de Deois flavopicta Stal (Hemiptera: Cercopidae), e IBCB 66 de $B$. bassiana (sensu lato), obtido de Hypothenemus hampei Ferrari (Coleoptera: Scolytidae). Os isolados foram mantidos em culturas estoques a $4^{\circ} \mathrm{C}$, na coleçáo do Laboratório de Microbiologia do Departamento de Produçáo Vegetal da Faculdade de Ciências Agrárias e Veterinárias (FCAV) da Universidade Estadual Paulista (UNESP). Para utilização nos ensaios foram cultivados em meio de batata, dextrose e ágar (BDA) a $27 \pm 0,5^{\circ} \mathrm{C}$ por 15 dias. Esporos removidos da superfície das colônias foram transferidos para tubos contendo mistura (1:1) de soluçáo de $\mathrm{NaCl}$ a $0,89 \%\left(\mathrm{p} \cdot \mathrm{v}^{-1}\right)$ e solução de Tween $80^{\circledR}$ a $0,1 \%\left(\mathrm{v} \cdot \mathrm{v}^{-1}\right)$. Após vigorosa agitação em agitador elétrico de tubos, as suspensôes foram padronizadas, com auxílio da câmara de Neubauer, na concentração de $10^{6}$ conídios. $\mathrm{mL}^{-1}$.

Para irradiar os conídios do isolado de $M$. anisopliae foram usadas as irradiâncias de 680, 750, 780 e $800 \mathrm{Wm}^{-2}$, na faixa espectral de 250 a $1150 \mathrm{~nm}$. Essas irradiâncias são valores médios ocorridos nos meses de novembro e dezembro de 2008, e janeiro e fevereiro de 2009 no município de Jaboticabal, São


Estação Agroclimatológica da FCAV/UNESP), meses esses geralmente de maior aplicaçáo do fungo para o controle da cigarrinha-da-raiz da cana-de-açúcar, no período de uma safra. Os conídios do isolado de $B$. bassiana foram submetidos às irradiâncias de 590, 570, 720, $640 \mathrm{Wm}^{-2}$, na faixa espectral já mencionada e correspondem, respectivamente, às irradiâncias médias ocorridas no município de Jaboticabal, São Paulo, nos meses que compóem as estações climáticas do outono, inverno, primavera e verão, tomando por base o período de 2008/2009 (dados fornecidos pela Estação Agroclimatológica da FCAV/UNESP), considerando a possibilidade de aplicaçáo do fungo em qualquer época do ano.

A irradiação foi feita utilizando um simulador solar Oriel ${ }^{\circ}$, modelo 68.820, Strafford, CT, USA, ajustado para emissão das irradiâncias desejadas, que foram medidas com auxílio de um radiômetro modelo 70.260, Strafford, CT, U.S.A. Em placas de Petri de $60 \mathrm{~mm}$ de diâmetro esterilizadas foram colocados $5 \mathrm{~mL}$ de suspensōes de conídios que foram expostas à luz do simulador, em cada uma das irradiâncias mencionadas, por períodos de 0, 30, 60, 120 e 180 minutos. Durante a irradiação as placas de análise ficaram dispostas dentro de outra 
placa de Petri de $150 \mathrm{~mm}$ de diâmetro contendo gelo, para evitar aquecimento pela radiação infravermelha. A irradiação foi realizada em temperatura ambiente $\left(26\right.$ a $\left.28^{\circ} \mathrm{C}\right)$. Para cada tratamento (irradiância e tempo de exposição) foram utilizadas três placas (repetiçóes) e para cada placa foi feita uma suspensão de conídios.

O ensaio de exposição à radiação UV foi realizado na câmara asséptica. Suspensões de conídios $(5 \mathrm{~mL})$ contidas em placas de Petri de $60 \mathrm{~mm}$ de diâmetro esterilizadas foram submetidas, por 0, 30, 60, 120, 180 e 240 segundos a uma lâmpada de raios ultravioleta germicida Toshiba de $30 \mathrm{~W}$. Essas placas, em grupo de 3 por tratamento, ficaram distantes $30 \mathrm{~cm}$ da lâmpada e foram submetidas aos raios ultravioleta na intensidade de $12 \mu \mathrm{W} / \mathrm{cm}^{2}$, medido com auxílio de um medidor digital Instrutherm, modelo MRV 201. A irradiação foi realizada em temperatura ambiente $\left(25\right.$ a $\left.28^{\circ} \mathrm{C}\right)$. Após a exposição dos conídios à luz do simulador solar ou aos raios UV, foi avaliada a germinação por meio do teste de viabilidade, usando lâminas de microscopia cobertas com fina camada de BDA, conforme metodologia descrita por FrANCISCO et al. (2006).

No ensaio para avaliar o efeito da temperatura foram utilizados:

- para o isolado de M. anisopliae as temperaturas de 19,5; 24,2 e $31,0^{\circ} \mathrm{C}$, que foram as médias das temperaturas mínima, mediana e máxima ocorridas nos meses de novembro e dezembro de 2008, e janeiro e fevereiro de 2009, no município de Jaboticabal, São Paulo (dados fornecidos pela Estação Agroclimatológica da FCAV/UNESP);

- para B. bassiana utilizou-se 19,$4 ; 20,8$ e $28,3^{\circ} \mathrm{C}$, e 18,7 ; 23,8 e $30,9^{\circ} \mathrm{C}$, que foram as médias de temperaturas mínima, mediana e máxima ocorridas em Jaboticabal, São Paulo, respectivamente, nas estaçóes climáticas do outono e inverno, e primavera e verão, tomando por base o período de março de 2008 a março de 2009 (dados fornecidos pela Estação Agroclimatológica da FCAV/UNESP).

Para avaliar a germinação dos conídios, lâminas de microscopia cobertas com fina camada de BDA foram previamente mantidas por 6 horas em estufa ajustada com uma das temperaturas antes mencionadas, para deixar o meio na temperatura desejada. Em seguida foi adicionada a suspensão de conídios e feita a avaliação pelo teste de viabilidade, conforme descrito por Francisco et al. (2006).

Em todos os experimentos empregou-se o delineamento inteiramente casualizado. A germinação de conídios expostos aos raios do simulador solar foi analisada segundo o esquema fatorial $5 \times 4$ ( 5 períodos e 4 intensidades de exposição), e a germinação de conídios expostos à radiação UV foi analisada segundo o esquema fatorial $6 \times 2$ (6 períodos e 2 espécies fúngicas). A análise da germinação dos conídios expostos a diferentes temperaturas foi feita segundo o esquema fatorial $3 \times 1$ (3 temperaturas e 1 espécie fúngica). Os dados foram submetidos à análise de variância pelo teste $\mathrm{F}$ e as médias comparadas pelo teste de Tukey a 5\% de significância. Para execuçáo das análises estatísticas usou-se o programa ESTAT (1997).

\section{RESULTADOS E DISCUSSÃO}

Os conídios do isolado de $M$. anisopliae foram severamente afetados pela exposiçấo aos raios do simulador solar. Conídios expostos por 30 minutos às irradiâncias de 680,750 e $800 \mathrm{Wm}^{-2}$, não tiveram a germinaçáo afetada, mas na irradiância de $780 \mathrm{Wm}^{-2}$ houve redução significativa da germinação a partir desse período de exposiçáo. Após 120 minutos de exposiçãa, a germinação foi menor do que $30 \%$ em todas as irradiâncias, mostrando que os conídios do isolado do fungo são pouco tolerantes à radiaçáo solar e que a exposiçấo por mais do que 30 minutos pode comprometer sua eficiência no controle (Tabela 1).

A irradiância teve menor interferência na germinação dos conídios do isolado do fungo do que o período de exposição. Nos períodos compreendidos entre 0 e 120 horas não houve reduçáo da germinação dos conídios expostos às diferentes irradiâncias. Quando a exposição foi feita por 180 minutos, verificou-se menor efeito deletério sobre os conídios na irradiância de $780 \mathrm{Wm}^{-2}$, resultando em maior germinação do que a verificada nas demais irradiâncias, embora os valores de germinação tenham sido bastante baixos nesse período de exposição (Tabela 1).

Tabela 1. Germinação (\%) de conídios do isolado de Metarhizium anisopliae expostos à radiação proveniente de um simulador solar em diferentes períodos e irradiâncias.

\begin{tabular}{|c|c|c|c|c|c|}
\hline \multirow{2}{*}{$\begin{array}{l}\text { Período de exposição } \\
\text { (minutos) }\end{array}$} & \multicolumn{4}{|c|}{ Irradiância $\left(\mathrm{Wm}^{-2}\right)$} & \multirow{2}{*}{ Teste F } \\
\hline & 680 & 750 & 780 & 800 & \\
\hline 0 & $99,87^{\text {Аa }}$ & $92,43^{\mathrm{Aa}}$ & $99,27^{\mathrm{Aa}}$ & $96,92^{\mathrm{Aa}}$ & $3,92 \mathrm{NS}$ \\
\hline 30 & $91,41^{\mathrm{Aa}}$ & $78,9^{\mathrm{Aa}}$ & $85,15^{\mathrm{Ba}}$ & $87,33^{\mathrm{Aba}}$ & $0,81 \mathrm{NS}$ \\
\hline 60 & $69,06^{\mathrm{BCa}}$ & $67,11^{\mathrm{Aa}}$ & $51,48^{\mathrm{Ca}}$ & $60,99^{\mathrm{Ba}}$ & $1,79 \mathrm{NS}$ \\
\hline 120 & $18,61^{\mathrm{BCDa}}$ & $29,33^{\mathrm{Ba}}$ & $29,42^{\mathrm{Da}}$ & $21,33^{\mathrm{ca}}$ & $0,49 \mathrm{NS}$ \\
\hline 180 & $4,43^{\mathrm{BCDab}}$ & $1,35^{\mathrm{Bb}}$ & $8,99^{\text {Еа }}$ & $1,13^{\mathrm{cb}}$ & $5,25^{*}$ \\
\hline Teste $\mathrm{F}$ & $100,7^{* *}$ & 31,33 ** & 920,33 ** & $35,87 * *$ & \\
\hline
\end{tabular}

Médias seguidas de mesma letra maiúscula na coluna ou minúscula na linha não diferem entre si pelo teste de Tukey a $5 \%$ de probabilidade; NS: não significativo; *significativo a $5 \%$ de probabilidade; **significativo a $1 \%$ de probabilidade. 
$\mathrm{O}$ isolado de $B$. bassiana mostrou grande sensibilidade à radiação do simulador solar, pois a redução da germinação variou entre 32 e 35\% já aos 30 minutos de exposição, considerando todas as irradiâncias. A exposição pelos demais períodos reduziu gradativamente a germinação em todas as irradiâncias, com menor efeito na irradiância de $750 \mathrm{Wm}^{-2}$, onde a germinação permaneceu constante entre 30 e 60 minutos de exposição (Tabela 2).

Analisando-se o efeito da irradiância em cada período de exposição verificou-se que a germinação dos conídios do isolado de $B$. bassiana foi afetada a partir de 60 minutos de exposição (Tabela 2).

Os isolados de ambos os entomopatógenos se mostraram bastante susceptíveis à radiação UV germicida (Tabela 3). Após 30 segundos de exposição à irradiação houve significativa redução da germinação $(38,2 \%)$ dos conídios do isolado de $M$. anisopliae, redução essa que se acentuou com o aumento do período de exposiçáo, até 240 segundos quando praticamente não restaram conídios germinados. $\mathrm{O}$ isolado de $B$. bassiana mostrou ser mais susceptível à radiação UV germicida do que o de $M$. anisopliae, pois com 30 segundos de exposição houve 65\% de redução da germinação, e essa redução se manteve com o aumento do período de exposição, encontrando-se apenas 2,6\% conídios germinados após 180 minutos de exposição.

A radiação solar tem papel importante na redução da viabilidade e morte de muitos esporos (CARLILE; WATKINSON, 1994). $\mathrm{Na}$ maioria dos estudos realizados com fungos filamentosos observou-se redução acentuada da viabilidade dos conídios após curtos períodos de exposição à radiação solar ou radiação UV. Conídios de B. bassiana, M. anisopliae e Metarhizium flavoviride Gams \& Rozsypal diminuiram acentuadamente a viabilidade com o aumento do período de exposiçáo à radiação UV incidente de simulador solar (MorLEY-DAVIEs et al., 1996). Conídios das linhagens ARSEF 23 e ARSEF 25 de $M$. anisopliae expostos diretamente à radiação solar por apenas 4 horas ficaram totalmente inativos (BRAGA et al., 2001c). A exposição de $B$. bassiana à radiação UV por 30 minutos ocasionou diminuição significativa no crescimento (CAGÁN; SverCel, 2001) e reduziu em $99,4 \%$ a viabilidade de conídios após 60 minutos de exposição (INGLIs et al., 1995). FERNANDES et al. (2007) observaram uma considerável redução na germinação de conídios do isolado Bb 19 de B. bassiana após 2 horas de exposição à radiação UV-B.

Neste estudo essa reduçáo de viabilidade foi verificada usando intensidades de radiação solar encontradas em condições naturais. A redução da germinação dos conídios decorre da necessidade das células consumirem energia para reparar danos ocasionados ao ácido nucléico e às proteínas, diminuindo a atividade celular para reparação dos sistemas lesados (Hirao et al., 2000; Zhou; Elledge, 2000).

A sensibilidade dos conídios à radiação solar e UV está relacionada com sua coloração (BRAGA et al., 2006; RANGEL et al., 2006). A pigmentação pode influenciar na tolerância de leveduras e fungos filamentosos à radiação (BRAGA et al., 2002). Conídios com tonalidade escura, laranja, creme e marrom podem ser tolerantes ao UV, devido aos carotenoides que os protegem contra as lesóes da radiação (Еıjк et al., 1979). A coloração clara influencia a inativação do conídio pela UV (IgnOffo; Garcia, 1992). Segundo esses autores, conídios de Aspergillus niger Tieghem com coloração muito escura foram mais tolerantes à radiação UV do que isolados de $M$. anisopliae, B. bassiana e Nomuraea rileyi (Farlow) Samson.

Tabela 3. Germinação (\%) de conídios dos isolados de Metarhizium anisopliae e de Beauveria bassiana expostos à radiação ultravioleta germicida emitida por lâmpada de 30 W.

\begin{tabular}{lcc|}
$\begin{array}{l}\text { Período de exposição } \\
\text { (segundos) }\end{array}$ & $\begin{array}{c}\text { Metarhizium } \\
\text { anisopliae }\end{array}$ & $\begin{array}{c}\text { Beauveria } \\
\text { bassiana }\end{array}$ \\
\hline 0 & $99,66^{\mathrm{A}}$ & $97,17^{\mathrm{A}}$ \\
\hline 30 & $61,54^{\mathrm{B}}$ & $33,9^{\mathrm{B}}$ \\
\hline 60 & $32,09^{\mathrm{C}}$ & $11,05^{\mathrm{C}}$ \\
\hline 120 & $12,79^{\mathrm{D}}$ & $5,36^{\mathrm{C}}$ \\
\hline 180 & $8,05^{\mathrm{DE}}$ & $2,62^{\mathrm{C}}$ \\
\hline 240 & $0,44^{\mathrm{E}}$ & 0,00 \\
\hline Teste F & $381,26^{* *}$ & $351,11^{\text {** }}$ \\
\hline C.V. $(\%)$ & 9,48 & 12.16 \\
\hline
\end{tabular}

Médias seguidas pela mesma letra maiúscula, na coluna, não diferem entre si pelo teste de Tukey a $5 \%$ de probabilidade; **Significativo a $1 \%$ de probabilidade; CV: Coeficiente de variação.

Tabela 2. Germinação (\%) de conídios do isolado de Beauveria bassiana expostos à radiação proveniente de um simulador solar em diferentes períodos e irradiâncias.

\begin{tabular}{|c|c|c|c|c|c|}
\hline \multirow{2}{*}{$\begin{array}{l}\text { Período de exposição } \\
\text { (minutos) }\end{array}$} & \multicolumn{4}{|c|}{ Irradiâncias $\left(\mathrm{Wm}^{-2}\right)$} & \multirow{2}{*}{ Teste F } \\
\hline & 590 & 520 & 640 & 750 & \\
\hline 0 & $91,67^{\mathrm{Aa}}$ & $91,19^{\mathrm{Aa}}$ & $90,69^{A a}$ & $92,54^{\mathrm{Aa}}$ & $0,85 \mathrm{NS}$ \\
\hline 30 & $61,99^{\mathrm{Ba}}$ & $62,36^{\mathrm{Ba}}$ & $55,5^{\mathrm{Ba}}$ & $60,75^{\text {Ba }}$ & $0,48 \mathrm{NS}$ \\
\hline 60 & $42,71^{\mathrm{cbc}}$ & $47,81^{\text {Bab }}$ & $25,79^{c c}$ & $60,46^{\mathrm{Ba}}$ & $3,89^{*}$ \\
\hline 120 & $22,31^{\mathrm{Dab}}$ & $17,92^{\mathrm{cab}}$ & $4,14^{\mathrm{Db}}$ & $26,86^{\mathrm{ca}}$ & $3,96 \mathrm{NS}$ \\
\hline 180 & $7,79^{\text {Eab }}$ & $10,12^{\mathrm{Cab}}$ & $1,02^{\mathrm{Db}}$ & $15,12^{\mathrm{Da}}$ & $4,04 \mathrm{NS}$ \\
\hline Teste F & $136,98^{* *}$ & 32,94 ** & $160,73^{* *}$ & $167,74^{* *}$ & \\
\hline
\end{tabular}

Médias seguidas de mesma letra maiúscula na coluna ou minúscula na linha não diferem entre si pelo teste de Tukey a 5\% de probabilidade; NS: não significativo; *significativo de $5 \%$ de probabilidade; **significativo a $1 \%$ de probabilidade. 
A importância da pigmentaçáo de conídios de $M$. anisopliae na sensibilidade à radiação solar foi estudada por BraGa et al. (2006), que verificaram que os conídios com pigmentação verde escuro são mais tolerantes. Metarhizium robertsii cultivado em meio PDAY sob luz visível contínua produziu conídios com tolerância à radiação UV-B quase duas vezes maior do que conídios produzidos pelo cultivo do fungo no mesmo meio, mas no escuro (RANGEL et al., 2011). Linhagens de Clonostachys rosea mostraram diferentes sensibilidades à radiação UV-B (Costa et al., 2012). Portanto, a sensibilidade de linhagens ou isolados fúngicos às radiaçóes solar e UV pode variar (RANGel et al., 2006). A diferença na sensibilidade dos isolados dos fungos utilizados neste trabalho à radiaçáo do simulador solar e UV pode ser atribuída à pigmentaçáo dos conídios, pois os de $M$. anisopliae têm coloração verde, devido à presença de pigmentos na parede, enquanto os conídios de B. bassiana têm coloração branca, provavelmente com pouco pigmento na parede.

A germinação dos conídios dos isolados de ambos os fungos foi afetada pela temperatura. Nas temperaturas de 24,2 e $31^{\circ} \mathrm{C}$, a germinaçáo dos conídios do isolado de $M$. anisopliae foi maior do que $98 \%$, enquanto que a $19,5^{\circ} \mathrm{C}$ houve drástica redução da germinação (Fig. 1A). Essas temperaturas correspondem às médias ambientais obtidas no período de novembro a fevereiro, meses em que normalmente mais ocorre a aplicaçáo de $M$. anisopliae para o controle da cigarrinha-da-raiz da cana-de-açúcar. Os resultados sugerem que os períodos mais frios do dia podem prejudicar a germinação do conídio, devendo ser evitados para aplicar o fungo no campo.

No período compreendido pelas estações climáticas da primavera e verão, onde predominaram temperaturas médias maiores do que $23,8^{\circ} \mathrm{C}$, houve maior germinaçáo dos conídios do isolado de B. bassiana (Fig. 1B). Nas estaçôes do outono e inverno, com temperaturas médias próximas a $20^{\circ} \mathrm{C}$, a germinação dos conídios foi drasticamente reduzida (Fig. 1C).

Temperatura e luz são fatores ambientais que regulam o desenvolvimento e processos fisiológicos da maioria dos organismos (BABitha et al., 2008). Segundo IsKandarov et al. (2006), a melhor germinação dos conídios de B. bassiana e $M$. anisopliae ocorre na faixa de 20 a $35^{\circ} \mathrm{C}$. Isolados de $M$. anisopliae var. anisopliae e $M$. anisopliae var. acridum obtidos nas latitudes $61^{\circ} \mathrm{N}$ a $54^{\circ} \mathrm{S}$ apresentaram grande variabilidade na tolerância à temperatura. Em geral, isolados provenientes de latitudes mais altas demonstraram maior suscetibilidade ao calor do que isolados obtidos perto da regiáo equatorial (Rangel et al., 2005). Os resultados deste estudo mostraram que a temperatura pode interferir no desempenho desses fungos como bioagentes de controle de pragas e que o emprego de B. bassiana no outono e inverno pode ser prejudicado, visto que temperaturas baixas dificultam a germinação dos conídios.
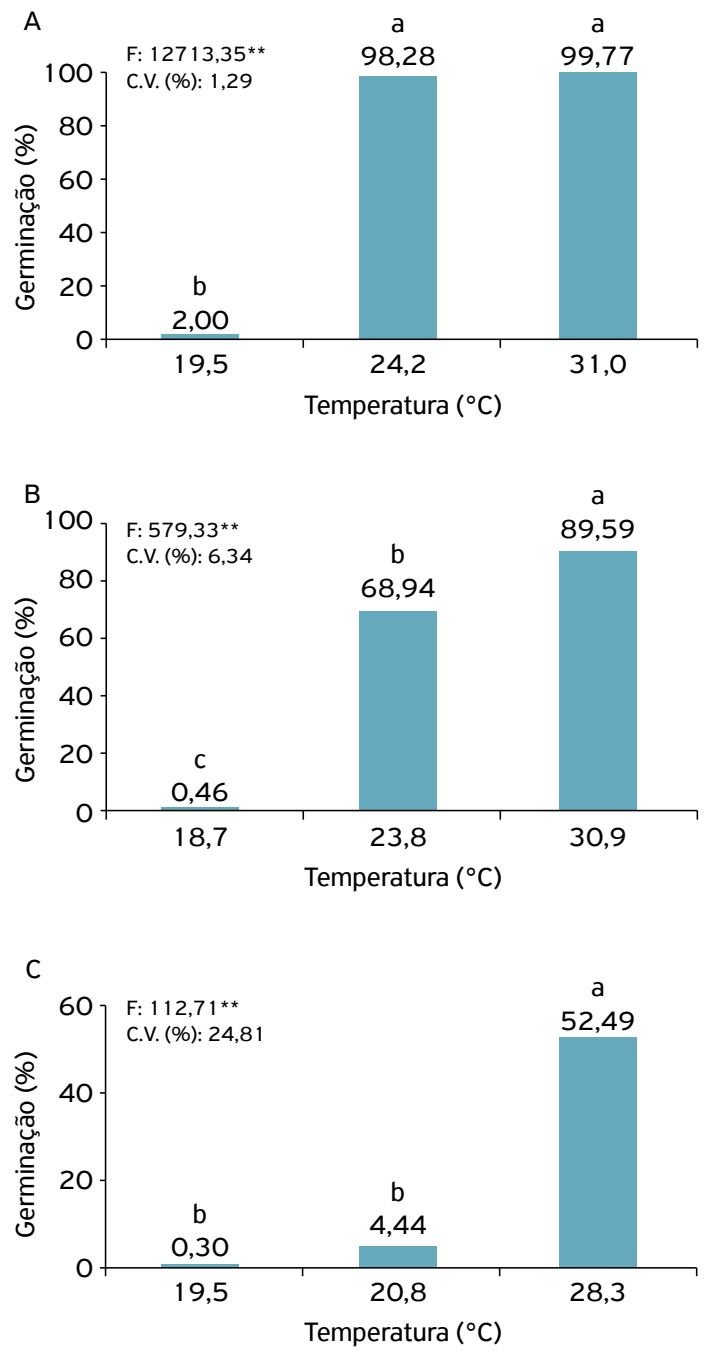

Médias acompanhadas de mesma letra minúscula não diferem entre si pelo teste de Tukey a $5 \%$ de probabilidade; ${ }^{* *}$ significativo a $1 \%$ de probabilidade; C.V.: coeficiente de variação.

Figura 1. Germinação de conídios do isolado de Metarhizium anisopliae expostos às temperaturas mínima, mediana e máxima ocorridas no município de Jaboticabal, São Paulo, no período de novembro de 2008 a fevereiro de 2009 (A), e do isolado de Beauveria bassiana exposto às mesmas temperaturas referentes às estações climáticas da primavera e verão (B), e do outono e inverno $(C)$, ocorridas no mesmo município, no período de março de 2008 a março de 2009.

\section{CONCLUSÕES}

Os isolados JAB 68 de M. anisopliae e IBCB 66 de B. bassiana são bastante sensíveis à exposição aos raios do simulador solar e UV, os quais promovem drástica redução da germinação de seus conídios. Os isolados são também afetados pela temperatura. Temperaturas entre 23,8 e $31^{\circ} \mathrm{C}$ favorecem a germinação dos conídios, enquanto temperaturas próximas a $20^{\circ} \mathrm{C}$ dificultam a germinação. 
ALMEIDA, J.E.M.; BATISTA FILHO, A.; SANTOS, A. Controle da cigarrinha-da-raiz da cana-de-açúcar, Mahanarva fimbriolata, com o fungo entomopatogênico Metarhizium anisopliae. STAB: Açúcar, Álcool e Subprodutos, Piracicaba, v.22, n.4, p.42-45, 2004.

ALVES, S.B. Fungos entomopatogênicos. In: ALVES, S.B. (Ed.) Controle microbiano de insetos. 2 ed. Piracicaba: FEALQ, 1998. Cap. 11 , p.289-381.

BABITHA, S.; CARVAHLO, J.C.; SOCCOL, C.R.; PANDEY, A. Effect of light on growth, pigment production and culture morphology of Monascus purpureus in solid-state fermentation. World Journal of Microbiology and Biotechnology, Dordrecht, v.24, n.11, p.26712675, 2008.

BATISTA FILHO, A.; MOINO JUNIOR, A.; ALVES, S.B. Recomendações para o uso de micoinseticidas destinados ao controle da broca-dabananeira. In: ALVES, L.F.A.; NEVES P.M.J.O.; FARIA, M.R. (Orgs.). Recomendações para utilização de fungos entomopatogênicos no controle de pragas. Piracicaba: CP2, 2010. p.21-24.

BRAGA, G.U.L.; FLINT, S.D.; MILLER, C.D.; ANDERSON, A.J.; ROBERTS, D.W. Variability in response to UV-B among species and strains of Metarhizium anisopliae isolates from sites at latitudes from $61^{\circ} \mathrm{N}$ to $54^{\circ} \mathrm{S}$. Journal of Invertebrate Pathology, San Diego, v.78, n.2, p.98-108, 2001a.

BRAGA, G.U.; FLINT, S.D.; MESSIAS, C.L.; ANDERSON, A.J.; ROBERTS, D.W. Effects of UV-B irradiance on conidia and germinants of the entomopathogenic Hyphomycete Metarhizium anisopliae: a study of reciprocity and recovery. Photochemistry and Photobiology, Amsterdam, v.73, n.2, p. $140-146,2001 \mathrm{~b}$.

BRAGA, G.U.; FLINT, S.D.; MILLER, C.D.; ANDERSON, A.J.; ROBERTS, D.W. Both solar UVA and UVB radiation impair conidial culturability and delay germination in the entomopathogenic fungus Metarhizium anisopliae. Photochemistry and Photobiology, Amsterdam, v.74, n.5, p.734-739, 2001 c.

BRAGA, G.U.; RANGEL, D.E.; FLINT, S.D.; MILLER, C.D.; ANDERSON, A.J.; ROBERTS, D.W. Damage and recovery from UV-B exposure in conidia of the entomopathogens Verticillium lecani and Aphanocladium album. Mycologia, New York, v.94, n.6, p.912920, 2002.

BRAGA, G.U.; RANGEL, D.E.; FLINT, S.D.; ANDERSON, A.J.; ROBERTS, D.W. Conidial pigmentation is important to tolerance against solar-simulated radiation in the entomopathogenic fungus Metarhizium anisopliae. Photochemistry and Photobiology, Amsterdam, v.82, n.2, p.418-422, 2006.

CAGÁN, L.; SVERCEL, M. The influence of ultraviolet light on pathogenicity of entomopathogenic fungus Beauveria bassiana (Balsamo) Vuillemin to the European corn borer, Ostrinia Nubilalis Hbn. (Lepidoptera: Crambidae). Journal of Central European Agriculture, Zagreb, v.2, n.3-4, p.228-232, 2001.

CARLILE, M.J.; WATKINSON, S.C. The fungi. San Diego: Academic Press, 1994. 428p.
COSTA, L.B.; RANGEL, D.E.; MORANDI, M.A.; BETTIOL, W. Impact of UV-B radiation on Clonostachys rosea germination and growth. World Journal of Microbiology and Biotechnology, Dordrecht, v.28, n.7, p.2497-2504, 2012.

DINARDO-MIRANDA, L.L.; VASCONCELOS, A.C.M.; FERREIRA, J.M.G.; GARCIA JUNIOR, C.A., COELHO, A.L.; GIL, M.A. Eficiência de Metarhizium anisiopliae (Metsch.) no controle de Mahanarva fimbriolata (Stal) (Hemiptera: Cercopidae) em cana-de-açúcar. Neotropical Entomology, Londrina, v.33, n.6, p.743-749, 2004.

ESTAT: Sistema para análises estatísticas. Versão 2.0. Jaboticabal: Faculdade de Ciências Agrárias e Veterinárias, 1997. Disponível em: <http://www.fcav.unesp.br/\#!/informatica/softwares/>. Acesso em: 10 Set. 2009.

EIJK, G.W.; MUMMERY, R.S.; ROEYMANS, H.J.; VALADON, L.R. Comparative study of carotenoids of Aschersonia aleyrodis and Aspergillus giganteus. Antonie van Leeuwenhoek, Amsterdam, v.45, n.3, p.417-422, 1979.

FERNANDES, E.K.; RANGEL, D.E.; MORAES, A.M.; BITTENCOURT, V.R.; ROBERTS, D.W. Variability in tolerance to UV-B radiation among Beauveria spp. isolates. Journal of Invertebrate Pathology, San Diego, v.96, n.3, p.237-243, 2007.

FRANCISCO, E.A.; MOCHI, D.A.; CORREIA, A.C.B.; MONTEIRO, A.C. Influence of culture media in viability test of conidia of entomopathogenic fungi. Ciência Rural, Santa Maria, v.36, n.4, p.1309-1312, 2006.

HIRAO, A.; Kong, Y.Y.; Matsuoka, S.; WAKEHAM, A.; RULAND, J.; Yoshida, H.; Liu, D.; Elledge, S.J.; MAK, T.W. DNA damageinduced activation of $\mathrm{p} 53$ by checkpoint kinase Chk2. Science, Washington, v.287, n.5459, p.1824-1827, 2000.

IGNOFFO, C.M.; GARCIA, C. Influence of conidial color on inactivation of several entomogenous fungi (Hyphomycetes) by simulated sunlight. Environmental Entomology, Lanham, v.2 1, n.4, p.913917, 1992

INGLIS, G.D.; DUKE, G.M.; GOETTEL, M.S.; KABALUK, J.T. Genetic diversity of Metarhizium anisopliae var. anisopliae in southwestern British Columbia. Journal of Invertebrate Pathology, San Diego, v.98, n. 1, p.101-113, 2008.

INGLIS, G.D.; GOETTEL, M.S.; JOHNSON, D.L. Influence of ultraviolet light protectants on persistence of the entomopathogenic fungus, Beauveria bassiana. Biological Control, Orlando, v.5, n.4, p.581590, 1995.

ISKANDAROV, U.S.; GUZALOVA, A.G.; DAVRANOV, K.D. Effects of nutrient medium composition and temperature on the germination of conidia and the entomopathogenic activity of the fungi Beauveria bassiana and Metarhizium anisopliae. Applied Biochemistry and Microbiology, New York, v.42, n. 1, p.72-76, 2006.

LANZA, L.M.; MONTEIRO, A.C.; MALHEIROS, E.B. Sensibilidade de Metarhizium anisopliae à temperatura e umidade em três tipos de solos. Ciência Rural, Santa Maria, v.39, n.1, p.6-12, 2009. 
MORLEY-DAVIES, J.; MOORE, D.; PRIOR, C. Screening of Metarhizium and Beauveria spp. conidia with exposure to simulated sunlight and a range of temperatures. Mycological Research, Cambridge, v. 100, n.1, p.31-38, 1996.

NEVES, P.M.J.O.; MOINO JUNIOR, A.; ALVES, S.B. Recomendações para o uso de micoinseticidas destinados ao controle da brocado-cafeeiro. In: ALVES, L.F.A.; NEVES, P.M.J.O.; FARIA, M.R. (Orgs.) Recomendações para utilização de fungos entomopatogênicos no controle de pragas. Piracicaba: CP2, 2010. p.25-28

NICHOLSON, W.L.; MUNAKATA, N.; HORNECK, G.; MELOSH, H.J.; SETLOW, P. Resistence of Bacillus endospores to extreme terrestrial end extraterrestrial environments. Microbiology and Molecular Biology Reviews, New York, v.64, n.3, p.548572, 2000.

RANGEL, D.E.N.; BRAGA, G.U.L.; ANDERSON, A.J.; ROBERTS, D.W. Variability in conidial thermotolerance of Metarhizium anisopliae isolates from different geographic origins. Journal of Invertebrate Pathology, San Diego, v.88, n.2, p. 1 16-125, 2005.

RANGEL, D.E.N.; BUTLER, M.J.; TORABINEJAD, J.; ANDERSON, A.J.; BRAGA, G.U.L.; DAY. A.W.; ROBERTS, D.W. Mutants and isolates of Metarhizium anisopliae are diverse in their relationships between conidial pigmentation and stress tolerance. Journal of Invertebrate Pathology, San Diego, v.93, n.3, p.170-182, 2006.

RANGEL, D.E.N.; FERNANDES, E.K.K.; BRAGA, G.U.L.; ROBERTS, D.W. Visible light during mycelial growth and conidiation of Metarhizium robertsii produces conidia with increased stress tolerance. FEMS Microbiology Letters, Oxford, v.315, n.2, p.81-86, 2011.

RATH, A.C. Ecology of entomopathogenic fungi in field soils. In: INTERNATIONAL COLLOQUIUM ON INVERTEBRATE PATHOLOGY AND MICROBIAL CONTROL, 8, Foz do Iguaçu, PR, 2002. Anais... Foz do Iguaçu: Society for Invertebrate Pathology, 2002. p.65-71.

REHNER, S.A. Ecology and evolution of fungal endophytes and their role against insects. In: VEGA, F.E.; BLACKWELL, M. (Eds.) Insect-fungal associations: ecology and evolution. Oxford: Oxford University Press, 2005. p.74-96.

WRAIGHT, S.P.; INGLIS, G.D.; GOETTEL, M.S. Fungi. In: LACEY, L.A.; KAYA, H.K. (Eds.) Field manual of techniques in invertebrate pathology. 2 ed. Dordrecht: Springer, 2007. Cap.4, p.223-248.

ZHOU, B.B.S.; ELLEDGE, S.J. The DNA damage response: putting checkpoints in perspective. Nature, London, v.408, n.6811, p.433-439, 2000 